\title{
Alignment of resonant optical cavities
}

\author{
Dana Z. Anderson
}

\begin{abstract}
When an input Gaussian beam is improperly aligned and mode-matched to a stable optical resonator, the electric field in the resonator couples to off-axis spatial eigenmodes. We show that a translation of the input axis or a mismatch of the beam waist to the resonator waist size causes a coupling of off-axis modes which is inphase with the input field. On the other hand, a tilt of the input beam or a mismatch of the beam waist position to cavity waist position couples to these modes in quadrature phase. We also propose a method to measure these coupling coefficients and thereby provide a means to align and mode-match a resonant optical cavity in real time.
\end{abstract}

\section{Introduction}

Proper alignment between an input laser beam and an optical cavity means exactly this: that the laser beam couples completely to the fundamental (longitudinal) spatial mode of the cavity and not at all to the higher-order (off-axis) spatial modes. We show that a transverse displacement and mismatch of waist size of the input beam with respect to the cavity axis and waist size give rise to inphase coupling to, respectively, the first- and second higher-order transverse modes of the cavity. These inphase coupling coefficients have been previously obtained by Rudiger et $a l^{1}$ in the context of suppressing laser beam fluctuation. On the other hand, angular misalignments and waist translations couple to these modes in quadrature phase.

By imposing onto a resonant nominally aligned input beam sidebands at the appropriate frequencies, one can measure these inphase and inquadrature components. In this way, the alignment and mode-matching errors are obtained directly and in real time, so our scheme can be part of a closed-loop system designed to maintain alignment. (We shall sometimes use the term alignment to mean angular orientation and transverse displacement of the input beam and sometimes to mean mode-matching as well as the former where there will be no ambiguity.) Furthermore, we show that changes between the cavity optical axis and a given reference point can be distinguished from changes between the laser beam path and that same reference. Therefore, both the cavity optical path and the laser beam path can

The author is with California Institute of Technology, Division of Physics, Mathematics \& Astronomy, Pasadena, California 91125. Received 12 April 1984.

0003-6935/84/172944-06\$02.00/0.

(C) 1984 Optical Society of America. be maintained with respect to a single chosen reference. Because the technique requires the laser frequency to be at a cavity resonant frequency, this scheme finds its niche most naturally in applications where the laser frequency is already to be maintained on a cavity resonance. Examples are cavity stabilized lasers, gravitational wave Fabry-Perot interferometers, and passive ring laser gyroscopes.

For purposes of our discussion we will consider the alignment of a two-mirrored optical cavity having spherical mirrors. The conclusions are easily generalized to cavities having more complex geometries, such as ring cavities employing elliptical focusing elements.

Section II obtains the coupling expressions for small misalignments of the input beam with respect to the cavity optical axis. Section III describes one possible implementation of the scheme. We close with a few comments on generalizing to more complicated geometries.

\section{Theory}

For background information on optical cavities the reader is directed to the review article by Kogelnik and $\mathrm{Li}^{2}$ For the most part the notation used here follows theirs.

The optical axis of a two-mirrored cavity is the line which intersects the center of curvatures of the two spherical mirrors of radius $R_{1}$ and $R_{2}$ as in Fig. $1 . \quad\left(R_{1}\right.$ and $R_{2}$ are both positive as drawn in the figure. $R_{1}$, for example, would be negative of the center of curvature if mirror 1 was to the left of mirror 1.) Associated with every stable optical cavity is a set of spatial eigenmodes which form a complete set. Physically, these eigenmodes describe electric field distributions which can propagate back and forth between the mirrors along the cavity axis with minimum diffraction losses and without 


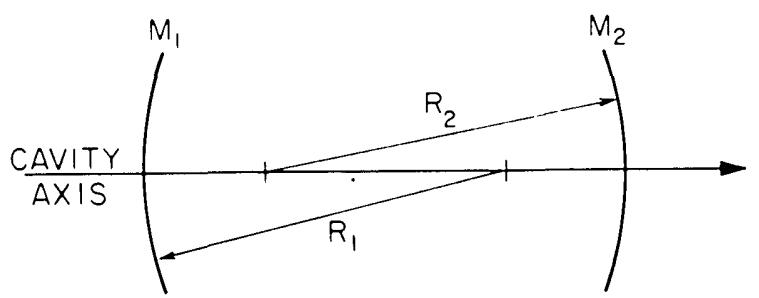

Fig. 1. Optical axis of a two-mirrored cavity is the line which intersects the centers of curvature of the two mirrors. This figure defines the sense of positive curvature for each mirror (after Kogelnik and $\left.\mathrm{Li}^{2}\right)$.

the distribution changing shape. A general input beam can be expanded and expressed as a linear combination of these eigenmodes. ${ }^{3}$ The expressions describing the eigenmodes throughout all space are rather messy. However, one can choose to expand the input beam anywhere along the cavity optical axis, and, therefore, we choose the point called the waist where the expressions assume their simplest form: at the waist the characteristic transverse dimension of the field is smallest, and the radius of curvature of the wave front is infinite. For a two-mirrored cavity there are six parameters which describe the input beam alignment and mode matching: two rotations, two transverse translations (alignment) and the waist axial position and size (mode matching), all measured at or from the cavity waist (which may actually be external to the cavity). With respect to the plane transverse to the optical axis, we will find it convenient to use a Cartesian coordinate system when dealing with the translations and rotations of the input beam and to use a polar coordinate system when dealing with waist size and waist position. We will consider the former first.

We discuss the transverse displacement and rotations in only one dimension, the $x$ dimension, because the $y$ dimension will have identical expressions. In one dimension, the normalized spatial eigenmodes are Hermite-Gaussians; the two lowest orders are of interest to us:

$$
\begin{aligned}
& U_{0}(x)=\left(\frac{2}{\pi x_{0}^{2}}\right)^{1 / 4} \exp \left[-\left(\frac{x}{x_{0}}\right)^{2}\right], \\
& U_{1}(x)=\left(\frac{2}{\pi x_{0}^{2}}\right)^{1 / 4} \frac{2 x}{x_{0}} \exp \left[-\left(\frac{x}{x_{0}}\right)^{2}\right],
\end{aligned}
$$

where $x_{0}$ is called the waist size. For a two-mirrored cavity of mirror spacing $d,{ }^{1}$

$$
x_{0}^{4}=\left(\frac{\lambda}{\pi}\right)^{2} \frac{d\left(R_{1}-d\right)\left(R_{2}-d\right)\left(R_{1}+R_{2}-d\right)}{\left(R_{1}+R_{2}-2 d\right)^{2}} .
$$

$U_{0}$ describes the fundamental mode, and $U_{1}$ is the first off-axis mode. We begin with the assumption that the input beam $\Psi(x)$ is Gaussian and aligned, so that $\Psi(x)$ $=A U_{0}(x)$ and then see what happens as it is misaligned. If we translate the input beam a small amount $a_{x}$, as in Fig. 2(a), $\Psi(x)$ becomes

$$
\begin{aligned}
\Psi(x) & =A U_{0}\left(x-a_{x}\right) \\
& =A\left(\frac{2}{\pi x_{0}^{2}}\right)^{1 / 4} \exp \left[-\frac{\left(x-a_{x}\right)^{2}}{x_{0}^{2}}\right] .
\end{aligned}
$$

The exponential can be expanded and if $a_{x} / x_{0} \ll 1$, terms of second order and higher in that fraction can be dropped:

$$
\Psi(x) \simeq A\left(\frac{2}{\pi x_{0}^{2}}\right)^{1 / 4}\left(1+2 a_{x} \frac{x}{x_{0}^{2}}\right) \exp \left[-\left(\frac{x}{x_{0}}\right)^{2}\right]
$$

or

$$
\Psi(x) \simeq A\left[U_{0}(x)+\frac{a_{x}}{x_{0}} U_{1}(x)\right] .
$$

Thus we see that a small transverse displacement of the input beam gives rise to a coupling of the first off-axis mode of the cavity.

What happens when the input beam is tilted with respect to the cavity axis? When viewed along the direction of travel, the input beam is a traveling wave whose phase along its axis of travel $z^{\prime}$ is given by $k z^{\prime}-$ $\omega t$, where $k$ is the wave number and $\omega$ is the angular frequency of the input beam. In general, the wave-front curvature along the axis of travel is spherical so that the off-axis phase is different from the on-axis phase. At the waist, however, the curvature is infinite so that the phase is constant along a transverse plane. We will assume here that the input beam waist matches the cavity waist; then suppose that the input beam is rotated about the cavity waist through a small angle $\alpha$ as shown in Fig. 2(b). As we project the magnitude of the input beam onto the cavity transverse plane, we see in Eq. (4) that it is no different from the input to first order in $\alpha_{x}$ :

$$
\begin{aligned}
|\Psi(x)| & =\left|\Psi\left(x^{\prime}\right)\right|\left(\cos \alpha_{x}\right)^{-1} \\
& \simeq\left|\Psi\left(x^{\prime}\right)\right|\left(1+\alpha_{x}^{2}+\text { higher order terms }\right) .
\end{aligned}
$$

However, the phase of the wave now varies along $x$ :
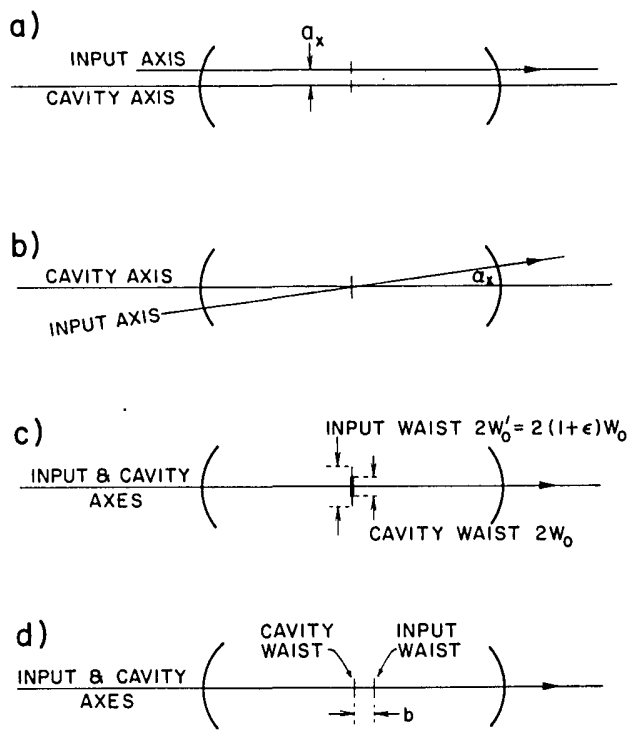

Fig. 2. Possible misalignments of the input axis with respect to the cavity axis: (a) transverse displacement in the $x$ direction; (b) tilt through an angle $a_{x} ;$ (c) waist size mismatch; and (d) axial waist displacement. Equivalent to (a) and (b) are transverse displacements and tilts in the $y$ dimension. 
Table I. Couplings due to Possible Misalignments of an Input Beam with Respect to the Cavily Fundamental Mode for a Two-Mirrored Cavity

\begin{tabular}{|c|c|c|c|c|c|}
\hline $\begin{array}{c}\text { Degree of } \\
\text { freedom }\end{array}$ & $\begin{array}{c}\text { Param- } \\
\text { eter }\end{array}$ & $\begin{array}{c}\text { Coupling } \\
\text { coeffi- } \\
\text { cient }\end{array}$ & Mode & $\begin{array}{c}\text { Phase } \\
\text { (deg) }\end{array}$ & Frequency \\
\hline $\begin{array}{c}\text { Transverse } \\
\text { position }\end{array}$ & $a_{\mu}$ & $a_{\mu} / w_{0}$ & $U_{1}(\mu)$ & 0 & $\nu_{0}$ \\
\hline $\begin{array}{l}\text { Angular tilt } \\
\text { (radius) }\end{array}$ & $a_{\mu}$ & $a_{\mu} \frac{\pi w_{0}}{\lambda}$ & $U_{1}(\mu)$ & 90 & $\nu_{0}$ \\
\hline Waist size & $w_{0}^{\prime}$ & $w_{0}^{\prime} / w_{0}-1$ & $V_{1}(r)$ & 0 & $2 \nu_{0}$ \\
\hline $\begin{array}{c}\text { Waist axial } \\
\text { position }\end{array}$ & $b$ & $\frac{\lambda b}{2 \pi w_{0}^{2}}$ & $V_{1}(r)$ & 90 & $2 \nu_{0}$ \\
\hline
\end{tabular}

Note: $\mu=x$ or $y . w_{0}$ is the cavity waist size, $\lambda$ is the source wavelength. $U_{1}(\mu)$ and $V_{1}(r)$ at the cavity waist are given in Eqs. $(1 \mathrm{~b})$ and (6b). $\nu_{0}$ is given in Eq. (11), where $c$ is the speed of light and $d$ is the mirror spacing. Positions are measured from the cavity waist.

$$
\varphi(x)=\left(\frac{2 \pi}{\lambda}\right) x \sin \alpha_{x} \simeq(2 \pi / \lambda) \alpha_{x} x
$$

or

$$
\Psi(x) \simeq A U_{0}(x) \exp \left(i \frac{2 \pi \alpha_{x} x}{\lambda}\right)
$$

The exponential may be expanded, and only the lowest-order terms are kept when $\left(2 \pi \alpha_{x} x_{0}\right) / \lambda \ll 1$. Physically this limit says that the tilt angle is smaller than the far-field divergence angle of the beam. Expanding, $\Psi(x)$ becomes

$$
\Psi(x) \simeq A U_{0}(x)\left(1+\frac{2 \pi i \alpha_{x} x_{0}}{\lambda}+\text { higher order terms }\right)
$$

or

$$
\Psi(x) \simeq A\left[U_{0}(x)+\pi i \frac{\alpha_{x} x_{0}}{\lambda} U_{1}(x)\right] .
$$

In words, a tilt couples into the first off-axis mode as does a transverse displacement but with a $90^{\circ}$ phase shift.

As mentioned above, to calculate coupling due to small mode-mismatching, it is more convenient to work in polar coordinates. The eigenmodes of the cavity are given by the generalized Laguerre polynomials $L_{l}^{p}$ weighted by a Gaussian. $\quad p$ and $l$ are the radial and angular mode numbers, respectively. We are interested here in the modes with no angular dependence and so set $l=0$. At the waist the two lowest-order radial modes are

$$
\begin{aligned}
& V_{0}(r)=\sqrt{\frac{2}{\pi}} \frac{1}{w_{0}} \exp \left(\frac{-r^{2}}{w_{0}^{2}}\right), \\
& V_{1}(r)=\sqrt{\frac{2}{\pi}} \frac{1}{w_{0}}\left(1-\frac{2 r^{2}}{w_{0}^{2}}\right) \exp \left(\frac{-r^{2}}{w_{0}^{2}}\right),
\end{aligned}
$$

where $r$ is the radial coordinate and the waist size has been renamed $w_{0}$.

This time suppose that the input beam is aligned and almost mode-matched except that the beam waist size is different from the cavity waist size by a small fraction $\varepsilon$, i.e., $w_{0}^{\prime}=w_{0}(1+\varepsilon)$ as in Fig. 2(c). Now

$$
\Psi(r)=A \sqrt{\frac{2}{\pi}} \frac{1}{w_{0}}(1+\varepsilon) \exp \left[-\frac{r^{2}}{w_{0}^{2}}(1+\varepsilon)^{2}\right] .
$$

Expanding the exponential and retaining terms to first order in $\varepsilon$

$$
\Psi(r) \simeq A\left[V_{0}(r)+\varepsilon V_{1}(r)\right] .
$$

Thus a small size mismatching excites a fraction $\varepsilon$ of the first higher-order radial mode.

The last case to be treated is the one in which the beam size is correctly matched, but the position of the waist is not [Fig. 2(d)]. This means that at the cavity waist the input beam has a finite radius of curvature. To describe what happens when the input waist is displaced a distance $b$ along the cavity axis $z$ from the cavity waist, one needs the expressions for the eigenmodes in a slightly more general form, describing how the modes evolve along the axis. Neglecting a common phase factor the two lowest-order modes are ${ }^{1}$

$$
\begin{aligned}
& V_{0}(r, z)=\sqrt{\frac{2}{\pi}} \frac{1}{w} \exp \left[-r^{2}\left(\frac{1}{w^{2}}+i \frac{\pi}{\lambda R}\right)\right], \\
& V_{1}(r, z)=\sqrt{\frac{2}{\pi}} \frac{1}{w}\left(1-2 \frac{r^{2}}{w^{2}}\right) \exp \left[-r^{2}\left(\frac{1}{w^{2}}-i \frac{\pi}{\lambda R}\right)\right] .
\end{aligned}
$$

$R$ is the radius of curvature of the wave front which varies along the cavity axis:

$$
R(z)=z\left[1+\left(\pi \frac{w_{0}^{2}}{\lambda z}\right)^{2}\right] .
$$

The spot size $w$ of the eigenmodes also varies along the cavity axis:

$$
w^{2}(z)=w_{0}^{2}\left[1+\left(\frac{\lambda z}{\pi w_{0}^{2}}\right)^{2}\right] .
$$

The distance $z$ along the cavity axis is measured from the cavity waist. At a short distance $b$ from the waist so that $\kappa \equiv\left[(\lambda b) /\left(\pi w_{0}^{2}\right)\right] \ll 1$, the curvature becomes $R(b)=\kappa^{-2}$. And, to first order in $\kappa$, the spot size is essentially that of the waist $w^{2}(b) \simeq w_{0}^{2}$. Therefore, the axially translated input beam at the cavity waist looks like

$$
\Psi(r, b)=A \sqrt{\frac{2}{\pi}} \frac{1}{w} \exp \left[\frac{-r^{2}}{w_{0}^{2}}(1-i \kappa)\right] .
$$

Expanding an exponential one final time gives the expression for the translated beam in terms of the eigenmodes: $\Psi(r, z)=A\left[V_{0}+(i / 2) \kappa V_{1}\right]$ or

$$
\Psi(r, z)=A\left(V_{0}+i \frac{\lambda b}{2 \pi w_{0}^{2}} V_{1}\right) .
$$

One last time, we see that an axial displacement of the input waist with respect to the cavity waist causes a coupling to the first higher-order radial eigenmode, this time in quadrature with the fundamental mode.

The results obtained so far are summarized in Table I. We conclude that any small misalignments cause a coupling into one of the lowest-order modes with a phase dependent upon the type of misalignment.

In general, the various spatial modes will have nondegenerate resonant frequencies. The frequency differences are characterized by a frequency $\nu_{0}$. The difference frequency between the fundamental mode 


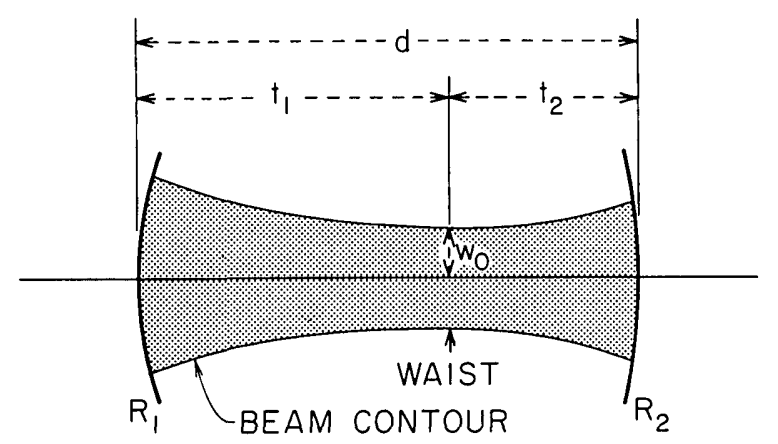

Fig. 3. Definition of waist distances $t_{1}$ and $t_{2}$; here both are shown positive (after Kogelnik and $\mathrm{Li}^{2}$ ).

$\left(\mathrm{TEM}_{00 q}\right)$ and an associated higher-order mode $\left(\mathrm{TEM}_{m n q}\right)$ or $\left(\mathrm{TEM}_{p l q}\right)$ are given by

$$
\delta \nu=k \nu_{0}
$$

where for a two-mirrored cavity of length $d$

$$
\nu_{0}=\frac{c}{2 d} \frac{1}{\pi} \cos ^{-1}\left[\left(1-\frac{d}{R_{1}}\right)\left(1-\frac{d}{R_{2}}\right)\right]^{1 / 2} .
$$

For $\mathrm{TEM}_{m n q}$ modes $k=m+n$ and for $\mathrm{TEM}_{p l q}$ modes $k=2 p+1$. The frequencies associated with the various misalignments are included in the table. The point to note is that misalignments cause a coupling to modes having $\delta \nu=\nu_{0}$, and mismatching couples to a mode having $\delta \nu=2 \nu_{0}$.

Obviously, a misalignment can occur not only by changing the input beam direction and so forth but also by changing the orientation or position of the cavity mirrors. The positions are usually fixed, but the orientations are often subject to drift. In general, a mirror tilt through an angle $\theta$ will cause a transverse displacement as well as a rotation of the optical axis, thereby giving rise to a linear combination of the inphase and inquadrature components of the first off-axis mode. Conversely, for each dimension, a proper linear combination of tilts from the two mirrors will give rise to a pure transverse displacement and a pure rotation of the optical axis. Call the mirror tilts $\theta_{1}$ and $\theta_{2}$. Recall that the optical axis is given by the line joining the centers of curvature of the mirrors. The distances $t_{1}$ and $t_{2}$ of the waist from the mirrors as shown in Fig. 3 are given by Eq. $(12)^{2}$ :

$$
\begin{aligned}
& t_{1}=\frac{d\left(R_{2}-d\right)}{R_{1}+R_{2}-2 d}, \\
& t_{2}=\frac{d\left(R_{1}-d\right)}{R_{1}+R_{2}-2 d} .
\end{aligned}
$$

Geometrical considerations show that the optical axis executes a pure transverse displacement when

$$
\theta_{2}=\frac{R_{1}}{R_{2}} \theta_{1}
$$

giving

$$
a=R_{1} \sin \Theta_{1}
$$

and a pure rotation about the waist when

$$
\sin \theta_{2}=\frac{\left(1-\frac{t_{2}}{R_{2}}\right)}{\left(1-\frac{t_{1}}{R_{1}}\right)} \sin \theta_{1}
$$

giving

$$
\sin \alpha=\frac{\sin \theta}{1-\frac{t_{1}}{R_{1}}} .
$$

In the above expressions $R_{1}$ and $R_{2}$ are positive if the centers of curvatures are as drawn in Fig. $3 ; t_{1}$ and $t_{2}$ are also positive as shown. Positive $\theta$ is counterclockwise.

As an example, considering a cavity composed of one flat and one curved mirror, a tilt of the flat mirror causes a pure rotation, a tilt of the curved mirror induces a pure displacement. Knowledge of the induced couplings caused by mirror tilts can be useful when the tilts are servo controlled.

We have described the kinds of coupling caused by small misalignments of the input beam with respect to the optical axis. We now discuss an embodiment of an alignment scheme based on these results.

\section{Instrumentation}

The proposed alignment scheme is shown in Fig. 4. It is supposed that the input beam is perhaps roughly, but at least nominally, aligned. The input frequency must be in resonance with the cavity which means that the source laser must be stabilized to the cavity length. Several authors have discussed techniques for locking a source laser frequency onto a cavity resonance (or vice versa). ${ }^{4,5}$ In most cases the alignment technique should be independent of the stabilizing apparatus; thus left out of the figure is the locking means. Imposed on the optical frequency is a sideband at the transverse mode frequency difference $\nu_{0}$ and/or $2 \nu_{0}$. (Actually one can put the sidebands at any of the first- or second-order off-axis mode frequencies $j c / 2 d+\nu_{0}$ and $j c / 2 d+2 \nu_{0}$.

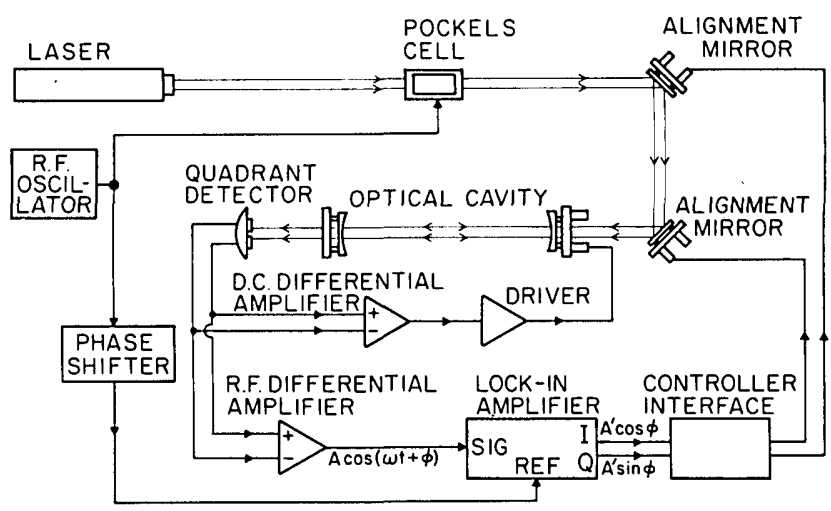

Fig. 4. Apparatus for controlling alignment. Instrumentation for controlling alignment in one dimension only is shown. Quadrant detector is fixed either physically or electronically to the left cavity mirror. The alignment signals are derived from the rf components of the detector outputs. 
Here $j$ is a positive or negative integer or zero, and $c$ is the speed of light.) In the figure this is done using a Pockels cell employed as a phase modulator driven by an oscillator at frequency $f=\nu_{0}$ and/or $f=2 \nu_{0}$. The Pockels cell creates other sidebands as well, but they will not affect the measurement in general.

What does the transmitted light from the cavity look like? If the input beam is perfectly aligned, no off-axis modes are excited, and none of the energy in the sidebands is transmitted because their frequencies are not resonant in the cavity fundamental mode. When the beam is misaligned, the coupling to the off-axis mode causes some of the sideband energy to be transmitted. For example, consider misalignments in the $x$ direction. If the amplitude of the fundamental mode is $E_{0}$ and the amplitude of the sideband at $\nu_{0}$ is $E_{1}$, the transmitted off-axis field is given by

$$
E_{1 t}=A_{1} E_{1} U_{1}(x) \exp \left\{-i\left[\left(\nu+\nu_{0}\right) t+\varphi_{1}+\varphi_{0}\right]\right\},
$$

where from Eqs. (3) and (5)

$$
\begin{aligned}
A_{1} & =\left[\left(a_{x} / x_{0}\right)^{2}+\left(\frac{\pi \alpha_{x} x_{0}}{\lambda}\right)^{2}\right]^{1 / 2}, \\
\varphi & =\tan ^{-1} \frac{\pi \alpha x_{0}^{2}}{\lambda a} .
\end{aligned}
$$

The constant phase factor $\varphi_{0}$ arises from the frequency difference between the fundamental and off-axis modes and the path length to the detector. The detector will see an intensity:

$$
\begin{aligned}
I(x)= & \left|E_{0} U_{0}(x)\right|^{2}+\left|A_{1} E_{1} U_{1}(x)\right|^{2} \\
& +2 A_{1} E_{0} E_{1} U_{0}(x) U_{1}(x) \cos \left(\nu_{0} t+\varphi+\varphi_{0}\right) .
\end{aligned}
$$

Because $U_{0}$ and $U_{1}$ are orthogonal over the transverse plane, if the detector intergrates over the entire plane, the interference term will disappear. Therefore, a split detector must be used so that the plane is cut into two halves. One then takes the difference of the two signals. The ac output of the differencing circuit will be proportional to the interference term: $V(t) \propto A_{1} E_{0} E_{1}$ $\cos \left(\nu_{0} t+\varphi+\varphi_{0}\right)$. The lockin amplifier then provides the inphase and inquadrature portions of this difference signal after nulling out the constant phase $\varphi_{0}$, which may now also have a contribution from the electronics. The outputs from the lockin amplifier are

$$
\begin{aligned}
V_{I} & \propto A_{1} E_{0} E_{1} \cos \varphi=\frac{a_{x}}{x_{0}} E_{0} E_{1}, \\
V_{Q} & \propto A_{1} E_{0} E_{1} \sin \varphi=\frac{\pi \alpha_{x} x_{0}}{\lambda} E_{0} E_{1},
\end{aligned}
$$

These can be used to control the input beam pointing or mirror positions.

In some applications it may be useful to orient one mirror with respect to the other. In this case, the input pointing can be controlled by the lockin output, the detector is fixed to the output mirror, and the orientation of the input mirror is adjusted whenever the optical axis drifts. This drift is detected by differencing the dc intensity on the two detector planes. By feeding this signal back to the input mirror, one forces the optical axis to intersect the center of the photodiode. When both dimensions $x$ and $y$ are to be controlled, it is necessary to use a quadrant detector. One obtains the required signals by taking the appropriate sums and differences of the detector elements. Also, in this case, two sets of lockin amplifiers are required unless some sort of multiplexing scheme is employed.

For mode-matching, all the same considerations made for the 1-D case above apply except that the sideband under consideration has frequency $f=2 \delta \nu_{0}$, and the detector must now be a two-element bull's-eye. If the amplitude of the sideband at $f=2 \nu_{0}$ is $E_{2}$, the detector sees an intensity:

$$
I(r)=\left|E_{0}\right|^{2}=\left|A_{2} E_{2}\right|^{2}+2 A_{2} E_{0} E_{2} V_{0}(r) V_{1}(r) \cos \left(2 \nu_{0} t+\varphi+\varphi_{0}^{\prime}\right),
$$

where from Eqs. (5) and (6)

$$
\begin{aligned}
A_{2} & =\left[\varepsilon^{2}+\left(\frac{\lambda b}{2 \pi w_{0}^{2}}\right)^{2}\right]^{1 / 2}, \\
\varphi & =\frac{\lambda b}{2 \pi w_{0 c}^{2}} .
\end{aligned}
$$

The outputs of the lockin amplifier will now be

$$
\begin{aligned}
V_{I} & \propto A_{2} E_{0} E_{2} \cos \varphi={ }_{\varepsilon} E_{0} E_{2}, \\
V_{Q} & \propto A_{2} E_{0} E_{2} \sin \varphi=\frac{\lambda b}{2 \pi w_{0}^{2}} E_{0} E_{2} .
\end{aligned}
$$

The control of the mode-matching is somewhat more complicated than for alignment since changing the beam waist size or axial position is usually not simply a matter of moving a single lens. ${ }^{6}$

In principle, to control all six input beam parameters, one could use a single polyelement detector such as a 4 $\times 4$ matrix rather than a quadrant and a bull's-eye. A polyelement diode would be even more useful in the case of a cavity having some arbitrary geometry. In general, a cavity can have eight degrees of freedom. For example, a ring cavity consisting of flat and spherical mirrors has different waist sizes for the $x$ and $y$ dimensions (perpendicular and parallel to the plane of a planar ring). ${ }^{7}$ Furthermore, there are two accompanying sets of resonant frequencies for the off-axis modes described by one-frequency spacing for the $x$ dimension and another for the $y$ dimension. Therefore, to detect all possible misalignments one may be required to have a total of four sidebands (two each for mode-matching and alignment in the $x$ and $y$ dimensions) and the same number of lockin amplifiers.

\section{Conclusions}

We have shown that misadjustments in the input beam alignment and mode matching give rise to off-axis mode couplings with a well-defined phase and amplitude. By imposing sidebands at the appropriate frequencies on a resonant beam of light, one can measure these couplings and thereby control the alignment and matching of the input beam with respect to the fundamental mode. We have also presented a possible implementation of a device using a two-mirrored cavity. By fixing the position of the detector relative to one mirror, it is possible to obtain information to control the second-mirror orientation as well as input alignment 
information, as was also discussed. Although the instrumentation is rather cumbersome, this technique has the advantage of supplying exactly the information that one usually wants (that is, the alignment of an input beam relative to the cavity fundamental mode), and it does so in real time.

The author owes special thanks to S. Whitcomb for providing valuable insights, to R. Spero and R. W. P. Drever for helpful discussions, and to K. S. Thorne for illuminating comments on the manuscript. This work was supported by the National Science Foundation grant PHY-82 04056.

\section{References}

1. A. Rudiger, R. Schilling, L. Schnupp, W. Winkler, H. Billing, and K. Maischberger, "A Mode Selector to Suppress Fluctuations in Laser Beam Geometry," Opt. Acta 28, 641 (1981).

2. H. Kogelnik and T. Li, "Laser Beams and Resonators," Appl. Opt. 5, 1550 (1966).

3. K. E. Oughstun, "On the Completeness of the Stationary Transverse Modes in an Optical Cavity," Opt. Commun. 42, 72 (1982).

4. Yu. V. Troitskii, "Optimization and Comparison of the Characteristics of Optical Interference Discriminators," Sov. J. Quanum. Electron. 8, 628 (May 1978).

5. See, for example, R. W. P. Drever, J. L. Hall, F. V. Kowalski, J. Hough, G. M. Ford, A. J. Munley, and H. Ward, "Laser Phase and Frequency Stabilization Using an Optical Resonator," Appl. Phys. B31, 97 (1983).

6. H. J. Baker, "Mode-Matching Techniques as an Aid to Laser Cavity Alignment," Opt. Acta 27, 897 (1979).

7. S. A. Collins, Jr., "Analysis of Optical Resonators Involving Focusing Elements," Appl. Opt. 3, 1263 (1964).

Meetings Calendar continued from page 2943

1984

November

4-8 2nd Int. Congr. on Advances in Non-impact Printing Technologies, Arlington H. Stark, Webster Res. Center, Xerox Corp., 800 Phillips Rd., W-114, Webster, N.Y. 14580

4-9 Cambridge Symp. on Optical \& Electro-Optical Eng. Cambridge SPIE, P.O. Box 10, Bellingham, Wash. 98227

4-9 Cambridge Symp. on Optical \& Electro-Optical Eng.: Intelligent Robots \& Computer Vision, Cambridge SPIE, P.O. Box 10, Bellingham, Wash. 98227

5-7 SESA Fall Conf.: Computer-Aided Testing \& Modal Analysis, Milwaukee SESA, 14 Fairfield Dr., Brookfield Ctr., Conn. 06805

5-8 Thermosense VII, Cambridge SPIE, P.O. Box 10, Bellingham, Wash. 98227

7-9 Micromachining \& Micropackaging of Transducers Mtg., Cleveland Case Western Reserve U., Semiconductor Chem. Transducer Resource, Electronics Design Center, Cleveland, Ohio 44106

8th Conf. on Application of Accelerators in Research \& Industry, Denton, Tex. J. Duggan, Physics Dept., $N$ Tex. State U., NT Box 5368, Denton, Tex. 76203

3rd Int. Congr. on Applications of Lasers \& ElectroOptics, Boston Laser Inst. of Am., 5151 Monroe St., Suite $118 \mathrm{~W}$, Toledo, Ohio 43623

12-16 Fiber \& Integrated Optics course, Ottawa Geo. Wash. U., Cont. Eng. Educ., Wash., D.C. 20052

Applications of Modern Optics course, Dallas Eng. Tech., Inc., P.O. Box 8859, Waco, Tex. 76714

Eastern Analytical Symp., New York T. Komentani, AT\&T Bell Labs., Rm. 1A-378, Murray Hill, N.J. 07074

13-16 Short Wavelength Laser Systems course, Albuquerque Eng. Tech., Inc., P.O. Box 8859, Waco, Tex. 76714

Applications-Oriented Approach to Artificial Intelligence course, Wash., D.C. Geo. Wash. U., Cont. Eng. Educ. Program, Wash., D.C. 20052

26-29 Energy Beam-Solid Interactions \& Transient Thermal Processing, Materials Res. Soc. Symposia, Boston $D$. Biegelson, Xerox Parc 3333, Coyote Hill Rd., Palo Alto, Calif. 94304

26-30 Materials Res. Soc. Fall Mtg., Boston J. Ballance, $M a$ terials Res. Soc., 9800 McKnight Rd. Suite 327, Pittsburgh, Pa. 15237

27-29 Advanced Photon \& Particle Techniques for Characterization of Defects \& Defect-Related Reactions in Solids, Materials Res. Soc. Symposia, Boston J. Roberto, Solid State Div., Oak Ridge Natl. Lab., P.O. Box X, Oak Ridge, Tenn. 37830

27-29 Laser Photochemical Processing \& Diagnostics, Materials Res. Soc. Symposia, Boston R. Osgood, Jr., Columbia U., 1312 SW Mudd Bldg., New York, N.Y. 10027

December

3-7 Fundamentals \& Applications of Lasers course, Albuquerque Laser Inst. of Am., 5151 Monroe St., Suite $118 \mathrm{~W}$, Toledo, Ohio 43623

11-13 Fiber Optics Communications course, Tempe Center for Professional Development, Coll. of Eng. \& Appl. Sci., Ariz. State U., Tempe, Ariz., 85287

1985

January

7-11 NSF Regional Conf.: Multivariate Estimation: A Synthesis of Bayesian \& Frequentist Approaches, U. Florida, Gainesville NSF, Math. Sciences, Wash., D.C. 20550

$7-18$

$9-11$
Optical Science \& Engineering course, Tucson P. Slater, P.O. Box 18667, Tucson, Ariz. 85287

Fiber Optics Workshop, Lake Buena Vista V. Amico, Coll. of Extended Studies, U. Central Fla., Orlando, Fla. 32816 